Z. Klin. Chem. Klin. Biochem.

10. Jg. 1972 , S. $555-561$

\title{
Eine gaschromatographische Methode zur Bestimmung der Sekretionsrate und Exkretionsrate von Aldosteron1)
}

\author{
Von O. Wörsdörfer ${ }^{2}$ ), G. DIEDRICHSEN und D. LOMMER \\ I. Mediziniscbe Klinik und Poliklinik der Jobannes Gutenberg-Universität, Mainz
}

(Eingegangen am 4. Februar/3. August 1972)

\begin{abstract}
Eine Methode zur gaschromatographischen Analyse von Aldosteron nach Säurehydrolysc im menschlichen Urin und deren Anwendung zur Bestimmung der Sekretionsrate und/oder Exkretionsrate des Hormones wird beschrieben. Parallelbestimmungen mit einer bewährten, klassischen Methode (Tetrazolium-Blau) ergaben übereinstimmende Ergebnisse. Die ermittelten Normalbereiche waren: Sekretionsrate $=144 \pm 86(2 \mathrm{~s}) \mu \mathrm{g} / \mathrm{d}(\mathrm{n}=35)$ und Exkretionsrate $=10,6 \pm 8,4(2 \mathrm{~s}) \mu \mathrm{g} / \mathrm{d}(\mathrm{n}=35)$. Die untere Nachweisgrenze der Methode lag bei $0,6 \mu \mathrm{g} / \mathrm{d}$, wenn die Hälfte eines 24-Stunden-Sammelurines aufgearbeitet wurde. Die Präzisionsprüfung ergab für die quantitative, gaschromatographische Analyse einen Variationskoeffizienten von $2,8 \%(n=90)$, für die gesamte Methode Variationskoeffizienten von $11,8 \%$ (Pool-Urin, $n=10$ ), bzw. 11,2\% (Doppelbestimmungen aus Patienten-Urinen, $n=48$ ). Die Proportionalitätsprüfung ergab zwischen ermittelten Aldosteronmengen und den eingesetzten Mengen eines Pool-Urines eine Korrelation von $r=0,999$. Die Richtigkeit der Methode wurde anhand der Wiederfindung von $11 \mu \mathrm{g}$ Aldosteron aus $1500 \mathrm{ml}$ Wasser $(n=6)$ geprüft. Sie betrug 10,23 $\pm 0,66(\mathrm{~s}) \mu \mathrm{g}$. Die gleichzeitige Bestimmung von Sekretions- und Exkretionsraten bei 122 Patienten crgab cine signifikante Korrelation ( $r=0,792$ ), obgleich 18 von 60 Patienten mit normaler Exkretionsrate eine erhöhte Sekretionsrate aufwiesen.
\end{abstract}

\section{A gaschromatographic method for the estimation of secretion and excretion rates of aldosterone}

A method for the analysis of aldosterone in acid hydrolyzed human urine, and its application for aldosterone secretion and/or excretion rate estimations are described. The results agreed with those of parallel estimations by means of a classical standard technique (blue tetrazolium). The normal ranges estimated were: secretion rate $=144 \pm 86(2 \mathrm{~s}) \mu \mathrm{g} / \mathrm{d}(\mathrm{n}=35)$, and excretion rate $=10.6 \pm 8.4(2 \mathrm{~s}) \mu \mathrm{g} / \mathrm{d}$ $(n=35)$. When one half of a complete $24 \mathrm{~h}$ urine was analyzed, the sensitivity of the method was $0.6 \mu \mathrm{g} / \mathrm{d}$. Examinations of the precision resulted in coefficients of variation of $2.8 \%(n=90)$ for the quantitative, gaschromatographic analysis, and of $11.8 \%$ (pool-urine, $n=10)$ or $11.2 \%$ (double estimations from patient urines, $n=48$ ) for the whole method. The correlation between the amounts of aldosterone found and the volumes of a pool urine analyzed was $t=0.999$. The accuracy of the method was examined by analyzing 6 portions of $1500 \mathrm{ml}$ water, each of which contained $11 \mu \mathrm{g}$ aldosterone. Results were $10.23 \pm 0.66$ (s) $\mu \mathrm{g}$.

Concomitant estimations of secretion rate and excretion rate in 122 patients resulted in a significant correlation $(r=0.792)$, although 18 of 60 patients with normal excretion rate exhibited increased secretion rate.

MERITS (1) berichtete 1962 über die Möglichkeit, Aldosteron nach Oxidation mit Perjodsäure als $11 \beta$ Hydroxy-3-keto-4-äthiensäure-18-al-18,11-hemiacetal-20, 18-lacton (im folgenden kurz als $\gamma$-Lacton bezeichnet) gaschromatographisch zu analysieren. Eine Reihe von Autoren (2-10) beschrieb in der Folgezeit entsprechende, gaschromatographische Methoden zur Bestimmung von Aldosteron in biologischen Flüssigkeiten.

In der vorliegenden Arbeit wird eine Methode zur Isolierung freien Aldosterons aus Urin nach Säurehydrolyse (Aldosteron-18-glucuronid + Aldosteron) und zu dessen quantitativ-gaschromatographischem Nachweis als $\gamma$-Lacton mittels Flammenionisationsdetektor beschrieben. Die Methode fand im Rahmen klinischer Untersuchungen praktische Anwendung in der Bestimmung der Aldosteronsekretions- und/oder -exkretionsraten nach dem Prinzip der Isotopenverdünnungsanalyse. Die Zuverlässigkeitskriterien der Methode wurden eingehend überprüft. Dazu wurden u. a. Paralleluntersuchungen mit einer bewährten, klassischen Technik (Tetrazolium-Blau) durchgeführt, die bereits seit 1963 (11) in der letzten Modifikation seit 1968 (12) in der Labordiagnostik angewandt wird.

\section{Methodik}

\section{Chemikalien}

D-Aldosteron: E. Merck. $\gamma$-Lacton: Eigene Herstellung durch Oxidation von D-Aldosteron mit Perjodsäure: Reinigung durch mehrfaches Umkristallisieren aus Methanol. $\left[1,2-{ }^{3} \mathrm{H}_{2}\right] \mathrm{D}$-Aldosteron $(30-50 \mathrm{Ci} / \mathrm{mmol})$ und $\left[4-{ }^{14} \mathrm{C}\right] \mathrm{D}-A$ ddosteron $(40-50 \mathrm{mCi} /$ mmol): NEN Chemicals. Reagenzien und Lösungsmittel („,zur Analyse"): E. Merck; alle Lösungsmittel wurden vor Gebrauch über $100 \mathrm{~cm}$ Glaskolonnen destilliert. Tetrazolium-Blau (3,3'Dianisol-bis-4,4'-(3,5-diphenyl)-tetrazoliumchlorid, p. a.): Serva.

\section{Geräte}

Photometer Eppendorf mit Cadmiumlampe und Filter Cd 509 nm: Netheler \& Hinz. Gaschromatograph Aerograph 2100 mit Flammenionisationsdetektor: Varian. Liquidscintillation Spectrometer Model 3380 mit Computer Model 544 und Radiochromatogram Scanner Model 7201: Packard Instruments.

Bestimmung der Aldosteronsekretions-und -exkretionsrate

Zur Bestimmung der Aldosteronsekretionsrate wurde den Probanden nach Entleerung der Blase zwischen 8 und 9 Uhr etwa

1) Mit Unterstutzung durch die Deutsche Forschungsgemeinschaft und durch das Bundesministerium für Bildung und Wissenschaft.

2) Auszug aus der Dissertation von Otto Wörsdörfer, Medizinische Fakultät der Johannes Gutenberg-Universität Mainz, 1972. 
$10 \mu \mathrm{Ci}\left[1,2-{ }^{3} \mathrm{H}_{2}\right] \mathrm{D}$-Aldosteron (gelöst in $1 \mathrm{ml}$ Athanol $+10 \mathrm{ml}$ physiologischer $\mathrm{NaCl}$-Lösung) quantitativ i. v. injiziert. Danach wurde der Urin über $72 \mathrm{~h}$ quantitativ gesammelt. Sollte aus dem Sammelurin gleichzeitig dic Aldosteronexkretionsrate bestimmt werden, so wurde dieser mit ctwa $0,05 \mu \mathrm{Ci}$ [4-14 C]D-Aldosteron als Verlustindikator versetzt. Die gesamte Urinmenge wurde in zwei 1/3-Portionen zur Doppelbestimmung nach der TetrazoliumBlau-Methode und zwei 1/6-Portionen zur Doppelbestimmung nach der gaschromatographischen Methode aufgeteilt. Zur Kontrolle der unspezifischen Blindwerte wurde mit jeder UrinDoppelbestimmung ein entsprechendes Volumen destillierten Wassers aufgearbeitet. Die Proben wurden mit konzentrierter $\mathrm{HCl}$ auf $\mathrm{pH} 1,1$ angesäuert und zur Hydrolyse des Aldosteron-18glucuronids über $24 \mathrm{~h}$ bei Raumtemperatur gehalten. Danach folgte eine dreimalige Extraktion mit jetveils $1 / 5$ des Probenvolumens an Chloroform. Die vereinigten Chloroformphasen wurden rasch mit jeweils $1 / 10$ ihres Volumens an eiskalter $0,1 \mathrm{~mol} / 1$ $\mathrm{NaOH}$ und anschließend mit destilliertem Wasser gewaschen. Das Lösungsmittel wurde bei $37^{\circ} \mathrm{C}$ im Rotationsverdampfer evaporiert. Zur ersten Chromatographie wurden die Rohextrakte mit wenig Chloroform auf dickes Chromatographiepapier (Macharey \& Nagel, MN 212; $10 \mathrm{~cm} \times 50 \mathrm{~cm}$ ) aufgetragen. Die Papierstreifen wurden durch Eintauchen in Essigsäure-iso-amylester:Methanol: Wasser $=40: 7: 3(\mathrm{v} / \mathrm{v})$ bis dicht an die Startzone imprägniert. Nach 10 bis 15 min wurden sie in einen dicht schließenden, mit Essigsäure-iso-amylester gesättigten Chromatographietank (Shandon) gebracht und anschließend über $16 \mathrm{~h}$ im Durchlauf mit wassergesättigtem Essigsäure-iso-amylester entwickelt (Raumtemperatur). Referenzsubstanz: Isatin. Der $R_{\text {Isatin-Wert des }}$ Aldosterons betrug im Mittel 0,45. Die exakte Lokalisation des Aldosterons erfolgte im Radiochromatogramm-Scanner. Nach Elution der Aldosteronbereiche mit Methanol und Evaporation des Lösungsmittels unterschied sich die weitere Aufarbeitung entsprechend der Art der quantitativen Auswertung (TetrazoliumBlau-Methode oder Gaschromatographie) wie folgt:

1. Tetrazolium-Blau-Methode

a) Rechromatographie auf dünnem Papier (Whatman Nr. 1; $5 \mathrm{~cm} \times 50 \mathrm{~cm}$ ) einfach absteigend $(4-5 \mathrm{~h})$ nach $12 \mathrm{~h} \mathrm{Äquili-}$

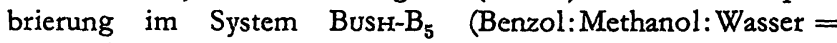
2:1:1 $\mathrm{v} / \mathrm{v}$ ). Elution der Aldosteronbereiche mit Methanol.

b) Acetylierung der sorgfältig getrockneten Eluate $(0,1 \mathrm{ml}$ wasserfreies Pyridin, 0,1 ml Essigsäureanhydrid, $12 \mathrm{~h}$ bei Raumtemperatur).

c) Chromatographische Isolierung des 18,21-Diacetoxy-aldosterons auf dünnem Papier (Whatman Nr. 1;5 cm $\times 50 \mathrm{~cm}$ ) im System Mesitylen:Methanol:Wasser $=9: 7: 3 \mathrm{v} / \mathrm{v}$. Nach Imprägnierung der Papierstreifen mit der Mesitylenphase Entwicklung mit der Methanol-Wasser-Phase im Durchlauf über $16 \mathrm{~h}$ (Raumtemperatur). Referenzsubstanz: Rodamin-B. Mittlerer $R_{\text {Rodamin-B-Wert }}$ des Aldosterondiacetates: 0,4 .

d) Rechromatographie auf Kieselgel-Dünnschicht (Macharey \& Nagel, Polygram SIL G-UV 254;2 cm $\times 20 \mathrm{~cm}$ Streifen auf $20 \times 20 \mathrm{~cm}^{2}$ Folien) im System Essigsäureäthylester: Aceton = 10:1 v/v einfach aufsteigend. Der mittlere $R_{F}$-Wert betrug 0,25 .

e) Die getrockneten Eluate der Aldosterondiacetat-Zonen und der entsprechenden Leerwert-Zonén wurden in $1 \mathrm{ml}$ Äthanol aufgenommen. Jeweils dreimal $0,05 \mathrm{ml}$ dieser Lösung wurden in Szintillationsmeßgläschen übergeführt, eingeengt, mit $10 \mathrm{ml}$ Szintillatorlösung (4 g 2,5-Diphenyloxazol, $50 \mathrm{mg}$ 1,4-bis-(2,5Phenyloxazolyl)-benzol, mit Toluol ad $1000 \mathrm{ml}$ ) aufgenommen und im Flüssigkeitsszintillationsspektrometer auf ihren Gehalt an ${ }^{3} \mathrm{H}$ - und/oder ${ }^{14} \mathrm{C}$-Radioaktivität analysiert. Je dreimal $0,2 \mathrm{ml}$ der Äthanollösung wurden mit $0,02 \mathrm{ml}$ Tetramethylammoniumhydroxidlösung ( $1 \mathrm{ml} 10 \mathrm{proz}$. wäßr. Tetramethylammoniumhydroxidlösung $+9 \mathrm{ml}$ Äthanol) und $0,02 \mathrm{ml} 0,5$ proz. äthanolischer Tetrazolium-Blau-Lösung versetzt. Nach exakt $25 \mathrm{~min}$ wurde die Reaktion durch Zugabe von $0,1 \mathrm{ml}$ Eisessig abgestoppt. Die Extinktion des gebildeten Formazans (13) wurde bei $509 \mathrm{~nm}\left(E_{\max }\right.$ bei $\left.510 \mathrm{~nm}\right)$ unter Verwendung von Mikroküvetten gegen Äthanol gemessen. Zu jeder Meßreihe wurde eine Eichkurve aus mindestens 5 Punkten erstellt, die stets linearen
Verlauf zeigte. Nach Leerwert-Korrektur wurden die jeweiligen Aldosteronmengen anhand der Eichkurve ermittelt.

2. Gaschromatographische Methode

a) Die Eluate wurden in je $0,2 \mathrm{ml}$ einer 1 proz. Lösung von $\mathrm{HJO}_{4}$ in Dioxan: Wasser $=1: 1$ aufgenommen. Nach $3 \mathrm{~h}$ bei Raumtemperatur wurde der Reaktionsansatz mit $2 \mathrm{ml}$ Wasser versetzt und mit $10 \mathrm{ml}$ Dichlormethan extrahiert. Der Extrakt wurde sorgfältig mit $2 \mathrm{ml} \quad 0,1 \mathrm{~mol} / \mathrm{l} \mathrm{NaOH}$ und anschließend bis zur Neutralität mit destilliertem Wasser gewaschen. Das Lösungsmittel wurde evaporiert.

b) Isolierung des $\gamma$-Lactons durch zweidimensionale Chromatographịe auf Kieselgel-Dünnschicht (Polygram SIL G-UV 254; $20 \mathrm{~cm} \times 20 \mathrm{~cm}$ ): 1. Lauf mit Hexan:Benzol: Essigsäureäthylester $=1: 4: 7(\mathrm{v} / \mathrm{v})$. Der $R_{\mathrm{F}}$-Wert des $\gamma$-Lactons betrug im Mittel 0,48. 2. Lauf senkrecht zur ersten Laufrichtung mit Methanol: Chloroform $=1: 9(\mathrm{v} / \mathrm{v})$. Der $R_{\mathbf{F}}$-Wert des $\gamma$-Lactons betrug im Mittel 0,35. Die $\gamma$-Lacton-Zonen (Lokalisation im kurzwelligen UV-Licht) und die Leerwert-Zonen wurden ausgeschnitten und mit je $2 \mathrm{ml}$ Äthanol eluiert. Nach Filtration durch Glasfritten G 4 wurde das Lösungsmittel evaporiert.

c) Zur Gaschromatographie des $\gamma$-Lactons wurde 3\% OV-1 auf 100-120 mesh Chromosorb-W (Varian) in einer $180 \mathrm{~cm} \times 0,3 \mathrm{~cm}$ U-förmigen Glassäule und $\mathrm{N}_{2}(55 \mathrm{ml} / \mathrm{min})$ als Trägergas verwendet. Das Flammengemisch des Detektors bestand aus $\mathrm{H}_{2}$ $(35 \mathrm{ml} / \mathrm{min})$ und Preßluft $(200 \mathrm{ml} / \mathrm{min})$. Die Temperatur des Säulenofens betrug $245^{\circ} \mathrm{C}$, die des Injektors und des Detektors $300^{\circ} \mathrm{C}$. Die Retentionszeit des $\gamma$-Lactons betrug unter diesen Bedingungen im Mittel 5 min. Vor jeder Meßreihe wurde die Trennsäule über Nacht unter Meßbedingungen konditioniert. Danach wurde bis zur konstanten Detektoranzeige $\gamma$-Lacton chromatographiert.

Das Eluat des Dünnschichtchromatogrammes wurde in 50 oder $100 \mu \mathrm{l}$ Essigsäureäthylester gelöst. Dreimal 10 oder $20 \mu \mathrm{l}$ dieser Löșung wurden zur Radioaktivitätsmessung entnommen (s. 1. e), und mindestens dreimal $3 \mu \mathrm{l}$ wurden zur quantitativen Bestimmung in die Trennsäule injiziert (Hamilton-Spritze $10 \mu \mathrm{l}$ ). $\mathrm{Zu}$ jeder Meßreihe wurde eine Eichkurve aus mindestens 5 Punkten erstellt, wobei jeder Eichpunkt aus dem mittleren Ergebnis von mindestens 3 Injektionen resultierte. Die Abbildungen 6 und 7 zeigen typische Eichkurven. Als $\mathrm{Ma} B$ der analysierten Aldosteronmenge diente der Flächeninhalt der Detektoranzeige über der Grundlinie. Voraussetzung für die Bewertung einer Analyse war es, daß bei der Chromatographie entsprechender Mengen des Leerwert-Eluates im Bereich des $\gamma$-Lactons keine Detektoranzeige erfolgte.

Die Sekretionsrate bzw. die Exkretionstate von Aldosteron wurde aus dem Verhältnis der $i$. v. injizierten, bzw. der dem Sammelurin zugesetzten Radioaktivitätsmenge $\mathrm{zu}$ der entsprechenden spezifischen Radioaktivität des isolierten Aldosterons berechnet:

Sekretionsrate $=$

$$
\frac{\text { injizierte }{ }^{3} \mathrm{H} \text {-Radioaktivität }(\mu \mathrm{Ci})}{\text { spezifische }{ }^{3} \mathrm{H} \text {-Radioaktivität }(\mu \mathrm{Ci} / \mu \mathrm{g}) \cdot 3}(\mu \mathrm{g} \text { Aldosteron/d })
$$

Exkretionsrate $=$

$$
\frac{\text { zugesetzte }{ }^{14} \mathrm{C}-\text { Radioaktivität }(\mu \mathrm{Ci})}{\text { spezifische }{ }^{14} \mathrm{C}-\text { Radioaktivität }(\mu \mathrm{Ci} / \mu \mathrm{g}) \cdot 3}(\mu \mathrm{g} \text { Aldosteron/d) }
$$

\section{Ergebnisse und Diskussion}

\section{Spezifität der Methode}

Als Kriterien der Spezifität der beschriebenen Methode können zunächst die Art der Isolierung des Aldosterons bzw. des $\gamma$-Lactons und die Art der quantitativen Analyse genannt werden. Die Alkaliwäsche des rohen Chloroformextraktes garantiert die weitgehende Entfernung der phenolischen Steroide und der Steroidsäuren. Die Papierchromatographie im Essigsäure-isoamylester-System eignet sich ganz besonders für die 
Isolierung des Aldosterons. Ein Großteil der Corticoidsteroide und Corticosteroidderivate (11-Desoxycorticosteron, Corticosteron, 11-Dehydrocorticosteron, 11-Desoxycortisol, Cortisol, Cortison und deren Tetrahydroderivate wurden geprüft) zeigt in diesem System höhere Wanderungsgeschwindigkeiten als Aldosteron. Besondere Bedeutung kommt der Alkaliwäsche des Dichlormethanextraktes nach der Perjodsäure-Oxidation zu. Die $\gamma$-Lactonbildung ist spezifisch für Aldosteron. Andere Steroide mit $\alpha$-Ketol-Seitenkette werden $\mathrm{zu}$ freien Äthiensäuren oxidiert. Diese werden durch die Natronlauge ausgewaschen, während das $\gamma$-Lacton in der organischen Phasc verbleibt. Die Gaschromatographie gewährleistet neben der Möglichkeit der quantitativen Auswertung einen zusätzlichen Selektionsschritt. Sie ermöglicht gleichzeitig eine qualitative Beurteilung des Meßwertes.

In diesem Zusammenhang muß allerdings darauf hingewiesen werden, da $\beta$ die beschriebene Methode nur dann brauchbare Resultate liefert, wenn während der gesamten Prozedur höchste Reinheitskriterien eingehalten werden. Erste Analysen unter "normalen" Laboratoriumsbedingungen ergaben außergewöhnlich hohe Werte für Sekretions- und Exkretionsraten, und im Wasser-Leerwert zeigte sich eine gaschromatographische Fraktion mit exakt der Retentionszeit des $\gamma$-Lactons (Abb. 1). Intensive Vorreinigung aller Lösungsmittel und Reagenzien führte nicht zur Lösung des Problems. Unterschiedliche Mengen der unspe-

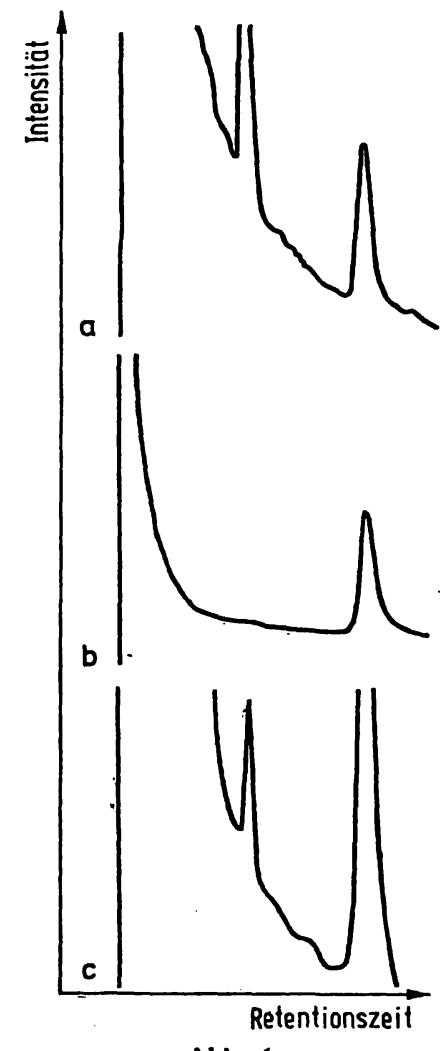

Abb. 1

Gaschromatographischer Nachweis einer unspezifischen Verunreinigung im Wasser-Leerwert mit den chromatographischen Eigenschaften des $\gamma$-Lactons. Elektrometerverstärkung: $2 \cdot 10^{-11} \mathrm{~A} / \mathrm{mV}$

a) Wasser-Leerwert, $3 \mu \mathrm{l}$ aus $1.00 \mu \mathrm{l}$ Endvolumen. b) $\gamma$-Lacton-Standard, $2 \mu \mathrm{l}=8 \mathrm{ng}$. c) Urin-Analysenprobe (SR 98), $3 \mu \mathrm{l}$ aus $100 \mu \mathrm{l}$

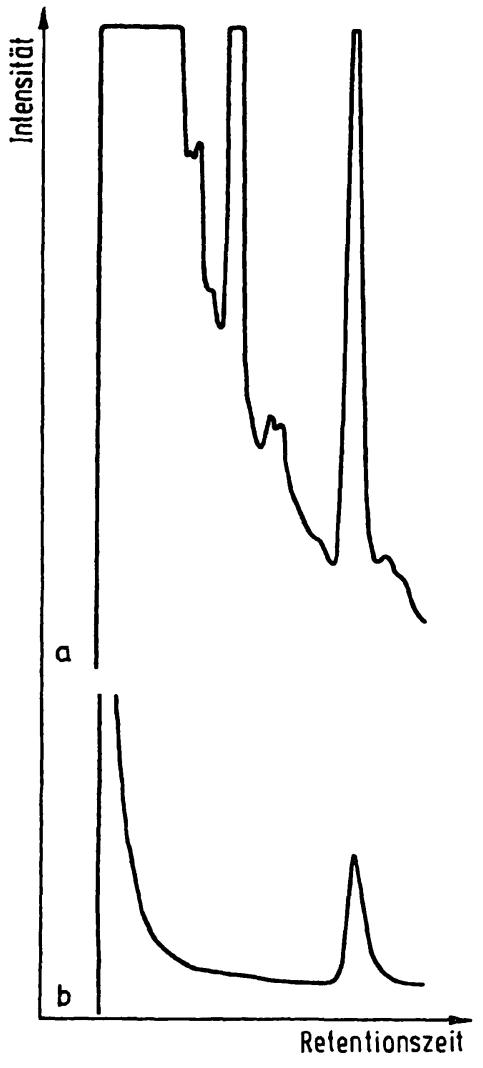

Abb. 2

Gaschromatographischer Nachweis der unspezifischen Verunreinigung (s. Abb. 1) im Essigsäureäthylester-Eluat eines menschlichen Fingerabdruckes. Elektrometerverstärkung: $2 \cdot 10^{-11} \mathrm{~A} / \mathrm{mV}$

a) Eluat des Fingerabdruckes. b) $\gamma$-Lacton-Standard, $2 \mu \mathrm{l}=8 \mathrm{ng}$

zifischen Fraktion in parallel untersuchten Wasserproben deuteten darauf hin, daß es sich nicht um eine systematische, sondern um eine zufällige Verunreinigung handelte. Wir chromatographierten deshalb das Essigsäureäthylester-Eluat eines Fingerabdruckes und fanden in dieser Lösung eine hohe Konzentration der Verunteinigung mit den gaschromatographischen Eigenschaften des $\gamma$-Lactons (Abb. 2). In der Folgezeit wurde jede Berührung von Chromatogrammen mit der bloßen Hand peinlichst vermieden, und alle Glasgeräte wurden kurz vor Gebrauch mit destilliertem Chloroform gespült. Beispiele für den Erfolg dieser Maßnahmen zeigen die Abbildungen 3 und 4: LeerwertProben zeigten fortan keinerlei Detektoranzeige im Bereich des $\gamma$-Lactons, und die Ergebnisse aus Urinanalysen lagen in plausiblen Größenordnungen. Bei Injektion einer Mischung aus Probe und Standard resultierte ein einheitlicher und symmetrischer Peak (Abb. 4).

Im Rahmen klinischer Routineuntersuchungen wurden 21 Aldosteronsekretionsraten (SR) und 26 Aldosteronexkretionsraten (ER) jeweils in Doppelbestimmung parallel nach der gaschromatographischen Methode (GLC) und nach der Tetrazolium-Blau-Methode (BT) bestimmt. Abbildung 5 zeigt den Vergleich der Ergebnisse. Mit

$\mathrm{SR}_{\mathrm{GLC}}=0,946 \cdot \mathrm{SR}_{\mathrm{BT}}-16,1 \quad(\mathrm{r}=0,981)$

und

$\mathrm{ER}_{\mathrm{GLC}}=0,966 \cdot \mathrm{ER}_{\mathrm{BT}}-0,1 \quad(\mathrm{r}=0,947)$ 


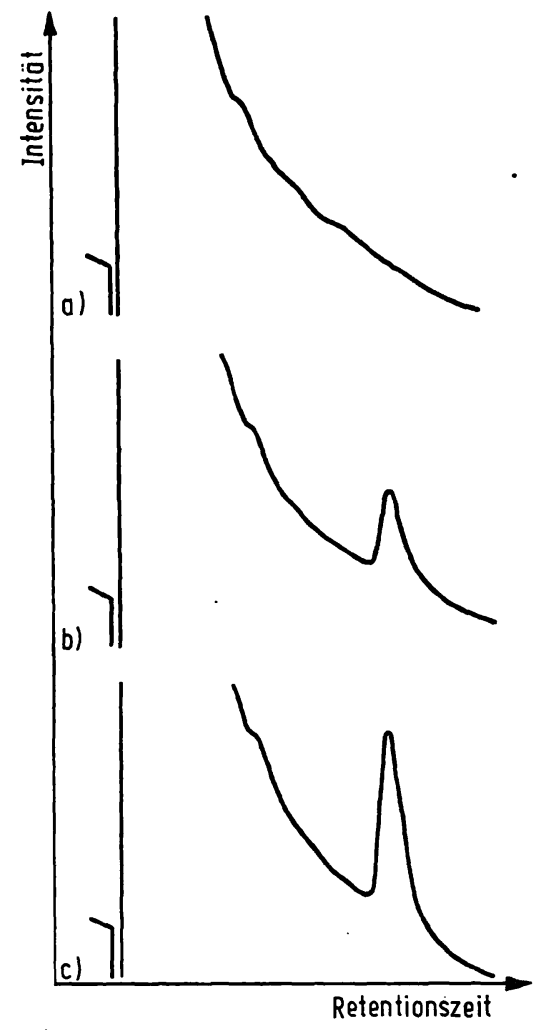

Abb. 3

Vermeidung der unspezifischen Verunreinigung durch erhöhte Sorgfalt bei der Probenaufbereitung. Elektrometerverstärkung: .

a) Wasser-Leerwert, $3 \mu \mathrm{l}$ aus $100 \mu \mathrm{l}$ Endvolumen. b) $\gamma$-Lacton-Standard, $1 \mu \mathrm{l}=4 \mathrm{ng}$. c) Urin-Analysenprobe (ER 78/I), $3 \mu \mathrm{l}$ aus $100 \mu \mathrm{l}$ Endvolumen

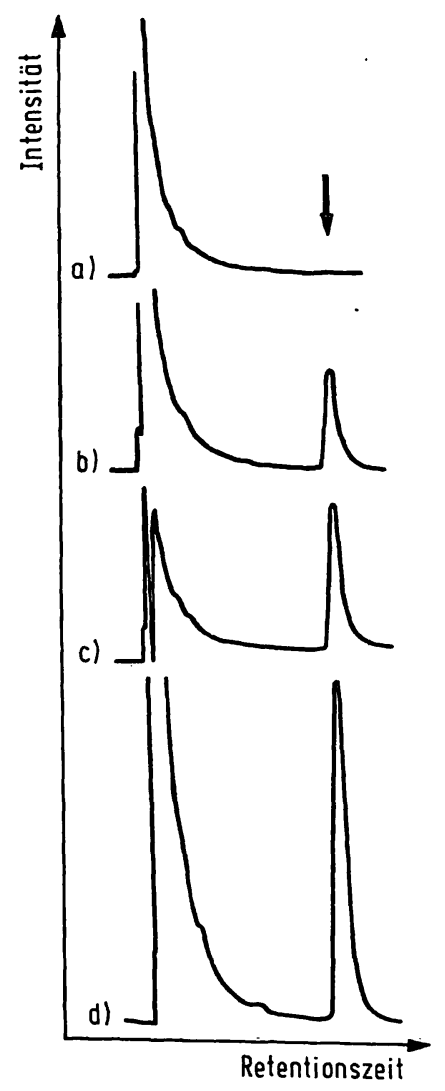

Abb. 4

Chromatographie bei Elektrometerverstärkung $10^{-10} \mathrm{~A} / \mathrm{mV}$. a) Wasser-Leerwert, $3 \mu \mathrm{l}$ aus $100 \mu \mathrm{l}$ Endvolumen. b) $\gamma$-Lacton-Stan$100 \mu \mathrm{l}$ Endvolumen. c) Urin-Analysenprobe (SR 192/I), $3 \mu 1$ aus SR $192 / 1$ ( $(3 \mu$ ) $)$-Lacton-Standard' $(3 \mu 1)$ und

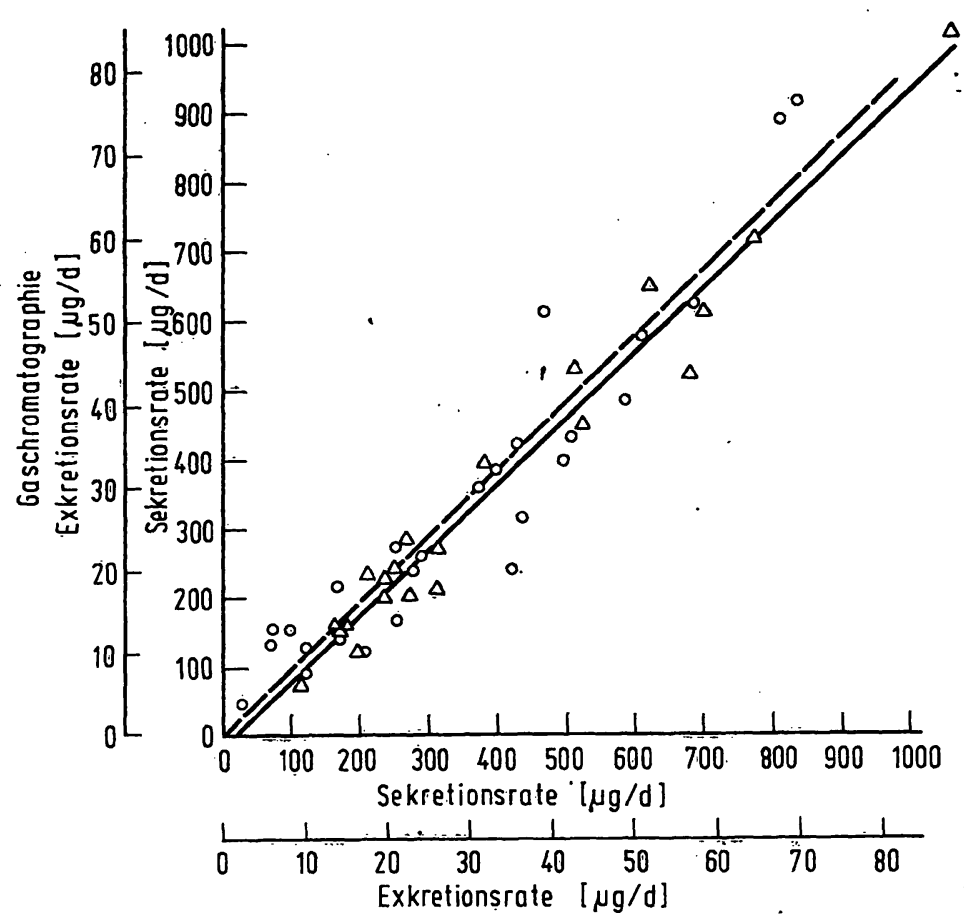

Tetro zolium - Blau - Methode

Abb. 5

Korrelation der Ergebnisse nach Parallelbestimmungen von Aldosteronsekretionsraten (SR, $\Delta,-\rightarrow$ ) und Aldosteronexkretionsraten (ER, o, - - -) mit der gaschromatographischen Methode (GLC) und mit der Tetrazolium-Blau-Methode (BT)

$E_{\text {G IC }}=0,966 \cdot \mathrm{ER}_{\mathrm{BT}}-0,1 ; \mathrm{r}=0,947(\mathrm{n}=26)$

ERGLC $=0,966 \cdot$ ERBT $_{\text {SR }}=0,1 ; r=0,947(\mathrm{r}=26)$
GLC $=0,946 \cdot \mathrm{SR}_{\mathrm{BT}}-16,1 ; \mathrm{r}=0,981(\mathrm{n}=21)$

ergab sich eine sehr gute Übereinstimmung bei hochsignifikanter Korrelation.

Für eine Kontrollgruppe von 17 weiblichen und 18 männlichen Patienten mittleren Alters, die keinerlei Anzeichen kardiovaskulärer, renaler, hepatischer oder endokriner Erkrankungen zeigten, fanden wir mit der gaschromatographischen Methode eine mittlere Aldosteronsekretionsrate von $144 \pm 86(2 \mathrm{~s}) \mu \mathrm{g} / \mathrm{d}$ (51 bis $203 \mu \mathrm{g} / \mathrm{d}$ ) und eine mittlere Aldosteronexkretionsrate von $10,6 \pm 8,4(2 \mathrm{~s}) \mu \mathrm{g} / \mathrm{d}(5-20 \mu \mathrm{g} / \mathrm{d})$. Diese Ergebnisse stimmen mit den Normalwerten überein, die von zahlreichen Arbeitsgruppen - unter Anwendung unterschiedlichster Methoden erstellt wurden (14).

\section{Empfindlichkeit der Methode}

Abbildung 6 zeigt eine typische Eichkurve bei einer empfindlichen Elektrometerverstärkung von $2 \cdot 10^{-11}$ $\mathrm{A} / \mathrm{mV}(100 \%$ Schreiberausschlag $=1 \mathrm{mV})$. Die untere Nachweisgrenze liegt danach bei einer etwa $3 \mathrm{ng}$ Aldosteron entsprechenden Menge $\gamma$-Lacton. Nimmt man ein üblicherweise eingesetztes Injektionsvolumen von $3 \mu \mathrm{l}$ aus $100 \mu \mathrm{l}$ Injektionslösung und stellt einen gesamten Aufarbeitungsverlust von $67 \%$. (Tab.) in Rechnung, so ließe sich bei Extraktion eines halben Tagesurines unter diesen Bedingungen noch eine Exkretionsrate von etwa $0,6 \mu \mathrm{g}$ bestimmen. Abbildung 3 zeigt typische Chromatogramme bei einer Elektrometerverstärkung von $2 \cdot 10^{-11} \mathrm{~A} / \mathrm{mV}$. Für die allgemeinen klinisch-diagnostischen Anforderungen genügt jedoch eine Elektrometerverstärkung von $1 \cdot 10^{-10}$ $\mathrm{A} / \mathrm{mV}$. Hierbei können $20 \mathrm{ng}$ Aldosteron noch gut gemessen werden. Eine entsprechende Eichkurve zeigt 


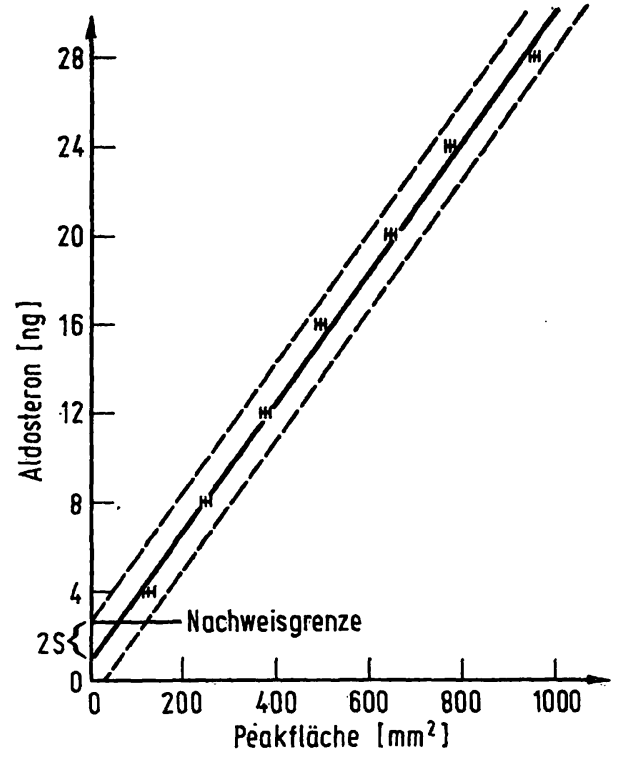

Abb. 6

Eichkurve für die gaschromatographische Aldosteronbestimmung als $\gamma$-Lacton bei Elektrometerverstärkung $2 \cdot 10^{-11} \mathrm{~A} / \mathrm{mV}$. Die $\gamma$-LactonKonzentration der Eichlösung (Essigsäureäthylester) entsprach $4 \mathrm{ng}$ Aldosteron $/ \mu$ ]. $\overline{\mathbf{x}} \pm \mathrm{s}$ (je 5 Injektionen) $0,029 \cdot \mathrm{mm}^{2}+0,94=\mathrm{ng}$ Aldosteron; $\mathrm{s}_{\mathbf{x}}=0,87 ; \mathrm{r}=0,998$ $+0,94=n_{g}$ Aldosteron; $s_{x}=0,87 ;$
Nachweisgrenze: $0,94+2 s_{x}=2,68 \mathrm{ng}$

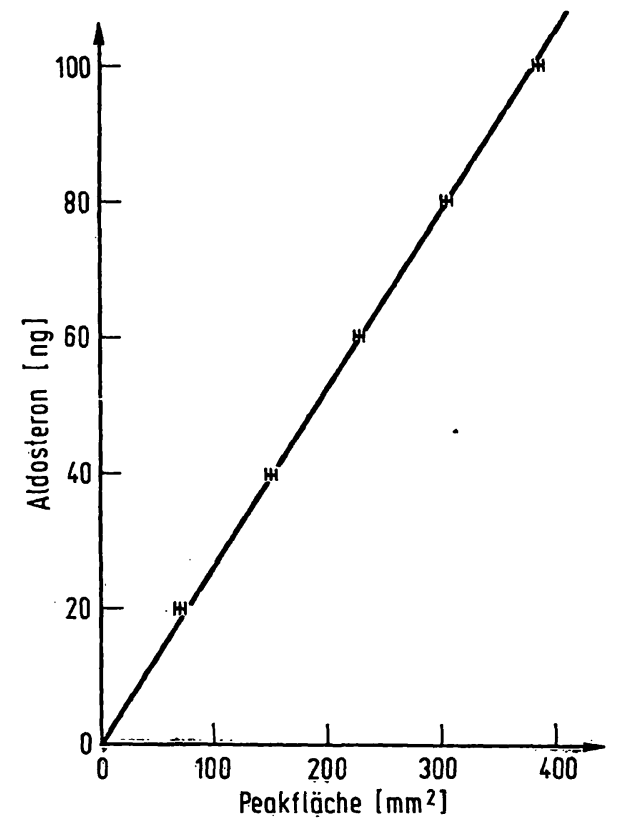

Abb. 7

Eichkurve für die gaschromatographische Aldosteronbestimmung als $\gamma$-Lacton bei Elektrometerverstärkung $10^{-10} \mathrm{~A} / \mathrm{mV}$. Die $\gamma$-Lacton Konzentration der Eichlösung (Essigsăureäthylester) entsprach 20ng Aldosteron $/ \mu \mathrm{l} . \overrightarrow{\mathrm{x}} \pm \mathrm{s}$ (je 5 Injektionen) Aldosteron/ $\mu \mathrm{l} \cdot \overline{\mathrm{x}} \pm \mathrm{s}$ (je 5 Injektionen)
$0,263 \cdot \mathrm{mm}^{2}+0,411=\mathrm{ng}$ Aldosteron $\mathrm{r}=0,997$

Tab. 1

Wiederfindung zugesetzten [4-1^C]D-Aldosterons aus $101000-\mathrm{ml}$ Aliquots eines Pool-Urines

\begin{tabular}{lcc}
\hline & $\begin{array}{c}\text { Mittlere } \\
\text { Wiederfindung } \\
\overline{\mathbf{x}} \pm \mathrm{s}\end{array}$ & Extremwerte \\
& $(\%)$ & $(\%)$ \\
\hline $\begin{array}{l}\text { Nach Hydrolyse, Extraktion } \\
\text { und Papierchromatographie }\end{array}$ & $75,7 \pm 4,8$ & $68,2-82,4$ \\
$\begin{array}{l}\text { Nach Perjodsäure-Oxidation } \\
\text { Nach Dünnschichtchromato- }\end{array}$ & $41,7 \pm 2,8$ & $38,4-44,6$ \\
graphie & $33,3 \pm 2,7$ & $29,6-36,8$ \\
\hline
\end{tabular}

Abbildung 7. In der Praxis läßt sich die Eichkurve mit 3 Punkten noch zuverlässig erstellen. Abbildung 4 zeigt typische Chromatogramme bei einer Elektroverstärkung von $1 \cdot 10^{-10} \mathrm{~A} / \mathrm{mV}$.

Präzision der Methode

1. Präzision der Gaschromatographie:

Aus 30 statistisch ausgewählten Dreifachinkjetionen von Meßproben aus Patienten-Urinen wurde der Variationskoeffizient für die entsprechende Detektoranzeige berechnet, indem das Ergebnis der Einzelinjektion in Prozent des entsprechenden Mittelwertes ausgedrückt wurde $(n=90)$. Der Variationskoeffizient betrug 2,8\%.

2. Präzision der gesamten Methode:

a) 10 1500-ml-Portionen eines Pool-Urines wurden unabhängig voneinander in Doppelbestimmungen analysiert. Das mittlere Ergebnis war 7,85 $\pm 0,93$ (s) $\mu \mathrm{g}$ Aldosteron $/ 1500 \mathrm{ml}$, entsprechend einem Variationskoeffizienten von $11,8 \%$.

b) Aus Doppelbestimmungen von 24 statistisch ausgewählten Urinanalysen wurde der Variationskoeffizient für die entsprechenden Einzelergebnisse berechnet, indem jedes Einzelergebnis in Prozent des entsprechenden Mittelwertes ausgedrückt wurde $(n=48)$. Der Variationskoeffizient betrug $11,2 \%$.

c) Sechs unterschiedliche Volumina eines Pool-Urines zwischen 500 und $2000 \mathrm{ml}$ wurden unabhängig voneinander analysiert. Die Korrelation zwischen den bestimmten. Aldosteronmengen und den eingesetzten Urinmengen ist in Abbildung 8 dargestellt. Sie ergab mit einem Korrelationskoeffizienten von 0,999 ausgezeichnete Proportionalität.

Richtigkeit der Methode

$11,0 \mu \mathrm{g}$ Aldosteron wurden in $1500 \mathrm{ml}$ Wasser gelöst. Sechs dieser Wasserproben wurden unabhängig von-

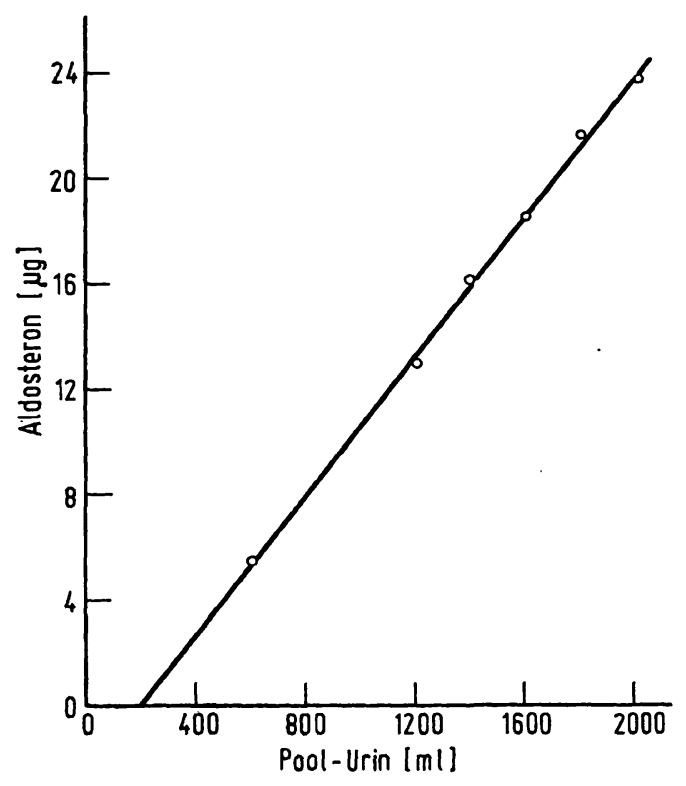

Abb. 8

Proportionalität der Ergebnisse bei Analyse unterschiedlicher Mengen eines Pool-Urines

$0,013 \cdot \mathrm{ml}-2,65=\mu \mathrm{g}$ Aldosteron; $\mathrm{r}=0,999$ 
einander in Doppelbestimmungen analysiert. Das mittlere Ergebnis betrug 10,23 $\pm 0,66$ (s) $\mu \mathrm{g}$, entsprechend einem Variationskoeffizienten von $6,5 \%$.

\section{Praktikabilität der Methode}

Wie vorangehend nachgewiesen wurde, besitzt die beschriebene Methode eine Zuverlässigkeit, die nach dem heutigen Standard für derartig schwierige Untersuchungen als sehr gut bezeichnet werden kann.

Der Aufwand für die Isolierung des Hormones konnte aufgrund der gaschromatographischen Bestimmung als $\gamma$-Lacton auf eine Papierchromatographie und eine zweidimensionale Dünnschichtchromatographie reduziert werden. Eine technische Hilfskraft führt zur Zeit pro Woche 10 Aldosteronsekretions- und/oder -exkretionsratenbestimmungen in Doppelbestimmungen durch.

Mit radioimmunologischen Methoden (15-18) können im Idealfall erheblich größere Analysenzahlen bewältigt werden. Leider ist es nur in seltenen Fällen gelungen (19), tatsächlich spezifische Antikörper gegen Aldosteron zu gewinnen. Nach dem heutigen Stand der Entwicklung setzt auch die radioimmunologische Technik einen erheblichen Aufwand an chromatographischer Vorreinigung vorraus und bietet deshalb vorerst nur unwesentliche Vorteile gegenüber gaschromatographischen Techniken. Bis zur Entwicklung höher spezifischer Antikörper werden gaschromatographische Methoden auch weiterhin, zumindest aber als Referenzmethoden, ihre volle Berechtigung beibehalten. Ein großer Vorteil der Gaschromatographie gegenüber dem Radioimmunoassay besteht in der Möglichkeit, die Zuverlässigkeit jeder einzelnen Analyse anhand von Lage und Form des Probenpeaks jederzeit zu beurteilen.

Korrelation zwischen Sekretionsrate (SR) und Exkretionsrate (ER)

Bei einem Kontrollkollektiv von 35 Patienten ohne Anzeichen kardiovaskulärer, renaler, hepatischer oder endokriner Erkrankungen, sowie bei 86 Patienten mit Verdacht auf Hyperaldosteronismus und einem Patienten nach subtotaler Adrenalektomie wurden gleichzeitig Sekretionsrate und Exkretionsrate bestimmt. Die Ergebnisse sind in Abbildung 9 zusammengefaßt. Zwischen Sekretionsrate und Exkretionsrate ergab sich ein $\mathrm{Zu}$ sammenhang gemäß $\mathrm{SR}=13,58 \cdot \mathrm{ER}+53,74 \mathrm{mit}$ einem Korrelationskoeffizienten von $r=0,792$. Damit besteht zwischen Sekretionsrate und Exkretionsrate eine signifikante Korrelation. Es fällt jedoch auf, daß 18 von 60 Fällen mit normaler Exkretionsrate (innerhalb des \pm 2 s-Bereiches der Kontrollgruppe) erhöhte Sekretionsraten zeigten $(30 \%)$. Von 40 Fällen mit normaler Sekretionsrate zeigte dagegen nur einer für die Exkretionsrate einen Wert, der geringfügig über der entsprechenden $+2 s$-Grenze lag.

Diese Befunde lassen sich, was zunächst naheliegend erscheint, nicht mit Radioaktivitätsverlusten bei der

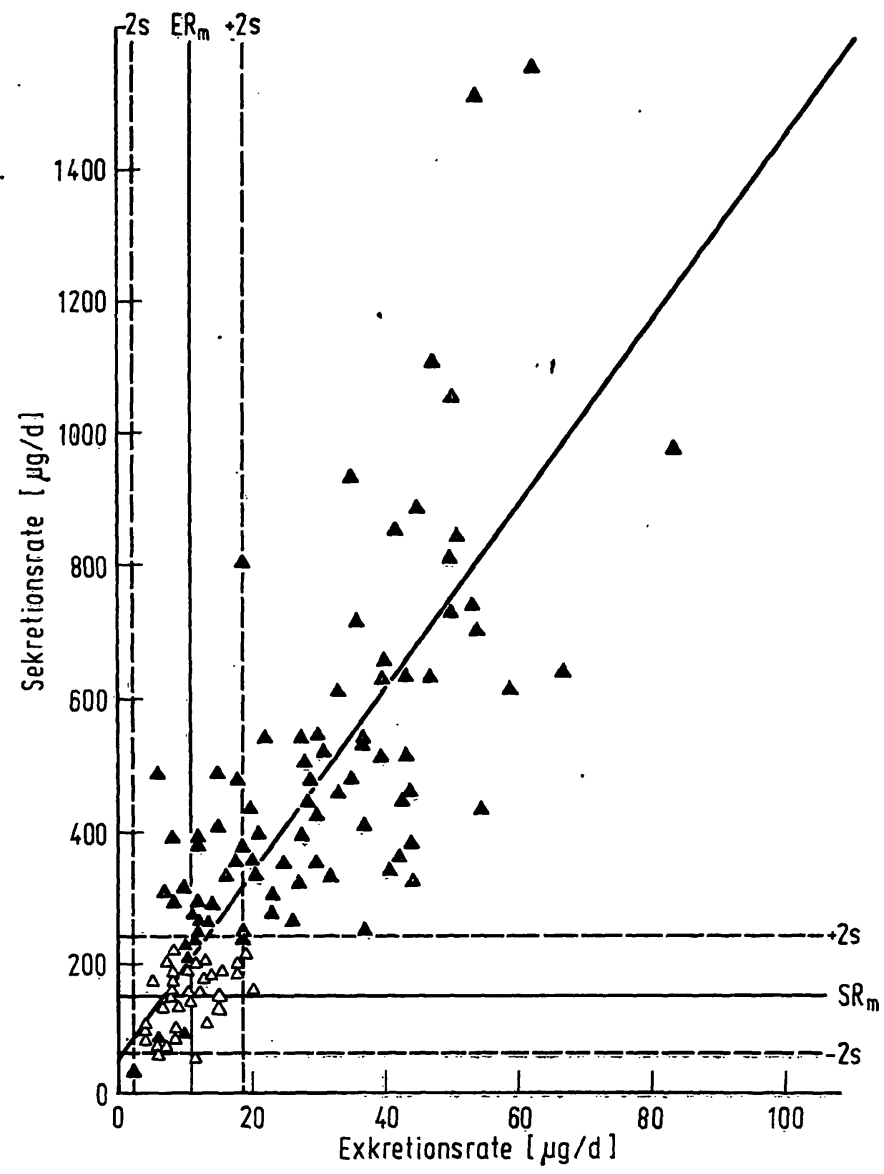

Abb. 9

Korrelation z̈wischen Aldosteronsekretionsraten (SR) und -exkretionsraten (ER). $\triangle:$ Kontrollgruppe; $\Delta$ : Patienten mit Verdacht auf Hyperaldosteronismus

$S R=13,58 \cdot E R+53,7 ; r=0,792(n=122)$

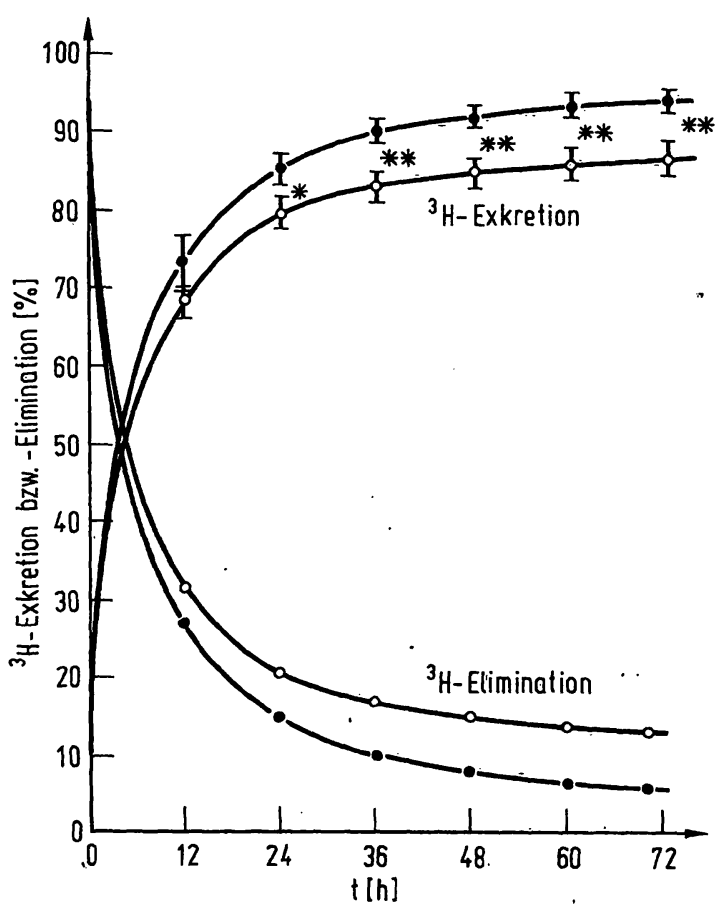

Abb. 10

Zeitlicher Verlauf der renalen ${ }^{3} \mathrm{H}-$ Exkretion nach i. v. Injektion von 'H-Aldosteron (100\%), sowie der 'H-Elimination aus dem Körper über die Nieren (Elimination $=100 \%-$ Exkretion) bei normaler

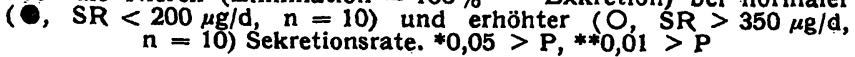


Endlicb wieder lieferbar - jetzt in 2., verbesserter Auflage!

\section{Georg Iwantscheff}

\section{Das Dithizon und seine Anwendung in der Mikro- und Spurenanalyse}

Es war eine glüdkliche Fügung, als 1925 Hellmuth Fischer das Diphenylthiocarbazon (Dithizon) in die analyrische Praxis einführte. Seitdem ist es das bedeutendste colorimetrische Extraktionsreagens geblieben - ausgezeichnet durch eine kaum zu übertreffende Reaktionsempfindlichkeit, Reaktionsbreite und zugleich Einstellmöglichkeit für hohe Trennschärfen.

Während die instrumentellen Verfahren (Spektralanalyse, Röntgenfluoreszens, Atomabsorption usw.) aufgrund ihrer Schnelligkeit die großen innerbetrieblichen Serienanalysen übernommen haben, ist das Dithizon für die Eichung, Kontrolle, Schiedsanalyse und für exakte kleine Serien die Methode der Wahl.
Mit Dithizon läßt sich der Großteil der Biogifte auf der Basis von Metallen und deren Organylverbindungen (Fungizide) im Spurenbereich erfassen, so daß dem Dithizon für den Umweltschutz eine bedeutende Schlüsselaufgabe zugewachsen ist. Neben dem Spurenanalytiker allgemein werden in Zukunft auch der Biologe und Arze zunehmend auf Dithizonmethoden zurückgreifen.

Fortschritte bei der Kontrolle der Reinheit von Solventien haben zur Stabilität der Reagenslösungen beigetragen.

Das Buch ist als Ratgeber für die analytische Praxis ausgelegt und als Grundlage für die Weiterentwicklung der reinen und der kombinierten (z. B. Chromatographie, Atomabsorption) Verfahren mit Dithizon.

Die jetzt vorliegende 2. Auflage des bekannten Standardwerkes wurde vom Autor selbst besorgt, und das Buch liegt nun in einer auf den neuesten Stand gebrachten Bearbeitung vor.

1972. 2., verbesserte Auflage. XVI, 330 Seiten mit 41 Abbildungen und

18 Tabellen.

Format $17 \times 24 \mathrm{~cm}$. Leinen DM 118,-

Ein Sonderprospekt steht zur Verfügung.

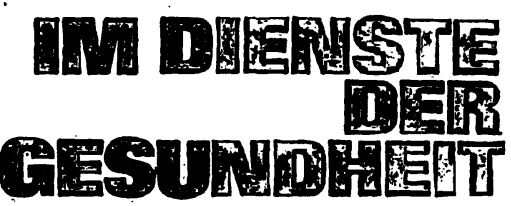

Als namhaftes Unternehmen der pharmazeutischen Industrie befassen wird uns mit der Entwicklung und Produktion immunbiologischer Präparate.

Im Rahmen des Ausbaues unserer Forschungsabteilung für Humanvirologie suchen wir

\section{Virologen, Mikrobiologen und Biochemiker}

die auf dem Gebiet der Virologie gearbeitet haben oder entsprechende Vorkenntnisse besitzen, als

\section{wissenschaftticine Mitarbeiter}

Ihnen bietet sich die Möglichkeit, die vielseitigen und interessanten Aufgaben bei der Entwicklung neuer Produkte kennenzulernen.

Durch Zusammenarbeit mit Instituten und Teilnahme an wissenschaftlichen Tagungen können Sie lhre Kenntnisse ergänzen und vertiefen.

Selbstverständlich ermöglichen wir Ihnen eine gründliche Einarbeitung in Thr künftiges Forschungsgebiet. Neben einer der qualifizierten Tätigkeit entsprechenden Dotierung gewähren wir alle sozialen Leistungen eines modernen Großunternehmens. Sie finden gutes Betriebsklima und einen jungen aufgeschlossenen Kollegenkreis.

Bei der Wohnungsbeschaffung in der landschaftlich reizvoll gelegenen Universitätsstadt Marburg sind wir Ihnen behilflich.

Sind Sie inferessiert, dann wenden Sie sich bitte kurz schriftlich an unsere Personalabteilung.

\section{Behringwerke Aktiengesellschaft}




\section{BIOCHIMIE}

\section{Edité par la Société de Chimie Biologique}

tel est le titre

sous lequel paraîtra à partir de 1971

le „BULLETIN DE LA SOCIÉTÉ DE CHIMIE BIOLOGIQUE““'

\section{SECRÉTARIAT}

de la Société de Chimie Biologique

J. P. EBEL, Secrétaire Général (Relations Extérieures)

R. PERLES, Secrétaire Général

\section{REDACTION}

F. GROS, Secrétaire scientifique

F. PERCHERON, Secrétaire à la Publication J. NUNEZ, Secrétaire à l'Information

Y. RAOUL, Secrétaire à l'Edition

SECRETARIAT et REDACTION: 4 Avenue de l'Observatoire, PARIS $6^{\circ}$

12 FASCICULES

ABONNEMENTS: FRANCE et ZONE FRANC: $150 \mathrm{fftcs}$ - BELGIQUE: 1.687,- frcs • AUTRES PAYS: $186,-$ ffrcs

MASSON et Cie, Editeurs · 120 Boulevard St Germain · PARIS 6éme

\section{Walter de Gruyter Berlin.New York}

\section{Karl Höll}

\section{Water}

Examination - Assessment - Conditioning

Chemistry - Bacteriology - Biology

With the collaboration of Helmut Peter and Dietrich Lüdemann

Translated from the 5 th German edition by Professor M.R. F. Ashworth,

Department of Organic Analysis,

University of the Saar, W. Germany

1972. XVIII + 389 pages.

With 24 diagrams in 129 figures.

Bound DM 76,-

ISBN 3110037289

\section{Karl Höll}

Wasser

Untersuchung - Beurteilung - Aufbereitung - Chemie - Bakteriologie Biologie

5., völlig neubearb. u. erw. Aufl. Mit 24 Abb. in 129 Einzeldarstellungen.

Gr.-Okt. XX, 423 S. 1970. Geb. DM 52,-

\section{Eckhart Buddecke}

\section{Grundriß der Biochemie}

Für Studierende der Medizin, Zahnmedizin und Naturwissenschaffen

3., neubearb. Aufl. Gr.-Okt. XXXII, $515 \mathrm{~S}$. Mit mehr als 400 Formeln, Tab. und Diagrammen. 1973. Plastik flexibel DM 29,50 ISBN 3110044315

(de Gruyter Lehrbuch)

\section{Günther Kraft Joseph Fischer \\ Indikation von Titrationen}

Okt. XII, 300 S. Mit 124 Abb. und 4 S. Tabellen. 1972. Plastik flexibel DM 58,- ISBN 3110016257 (Arbeitsmethoden der modernen Naturwissenschaften) 
Urinsammlung erklären, da in allen Fällen mehr als $80 \%$ der injizierten Radioaktivität im Sammelurin wiedergefunden wurden.

Untersuchungen über den zeitlichen Verlauf der Ausscheidung der gesamten Radioaktivität bei $10 \mathrm{~Pa}$ tienten mit Sekretionsrate $<200 \mu \mathrm{g} / \mathrm{d}$ und $10 \mathrm{~Pa}$ tienten mit Sekretionsrate $>350 \mu \mathrm{g} / \mathrm{d}$ ergaben eine signifikant langsamere Ausscheidung bei den Patienten mit erhöhter Sekretionsrate (Abb. 10). Die Analyse der mittleren Eliminationskurven (zusammengesetzte Exponentialfunktionen) in halblogarithmischer Darstellung (Abb. 11) ergab jeweils mindestens drei Exkretionsfraktionen für das injizierte ${ }^{3} \mathrm{H}$-Aldosteron.
Dabei deutete sich bei Patienten mit erhöhter Sekretionsrate eine relative Verkleinerung der am schnellsten eliminierten Fraktion an. Diese rasch eliminierte Fraktion entspricht mit großer Wahrschcinlichkeit der für die Analyse des Aldosteron-18-glucuronides verwendeten säurelabilen Fraktion (20). Die festgestellte Divergenz zwischen normaler Exkretionsrate und erhöhter Sekretionsrate könnte demnach aus einer Veränderung im Muster der ausgeschiedenen Aldosteronmetaboliten bei erhöhter Aldosteron-Plasmakonzentration resultieren. Die endgültige Klärung dieser Fragestellung bedarf jedoch zweifellos weiterer eingehender Untersuchungen.
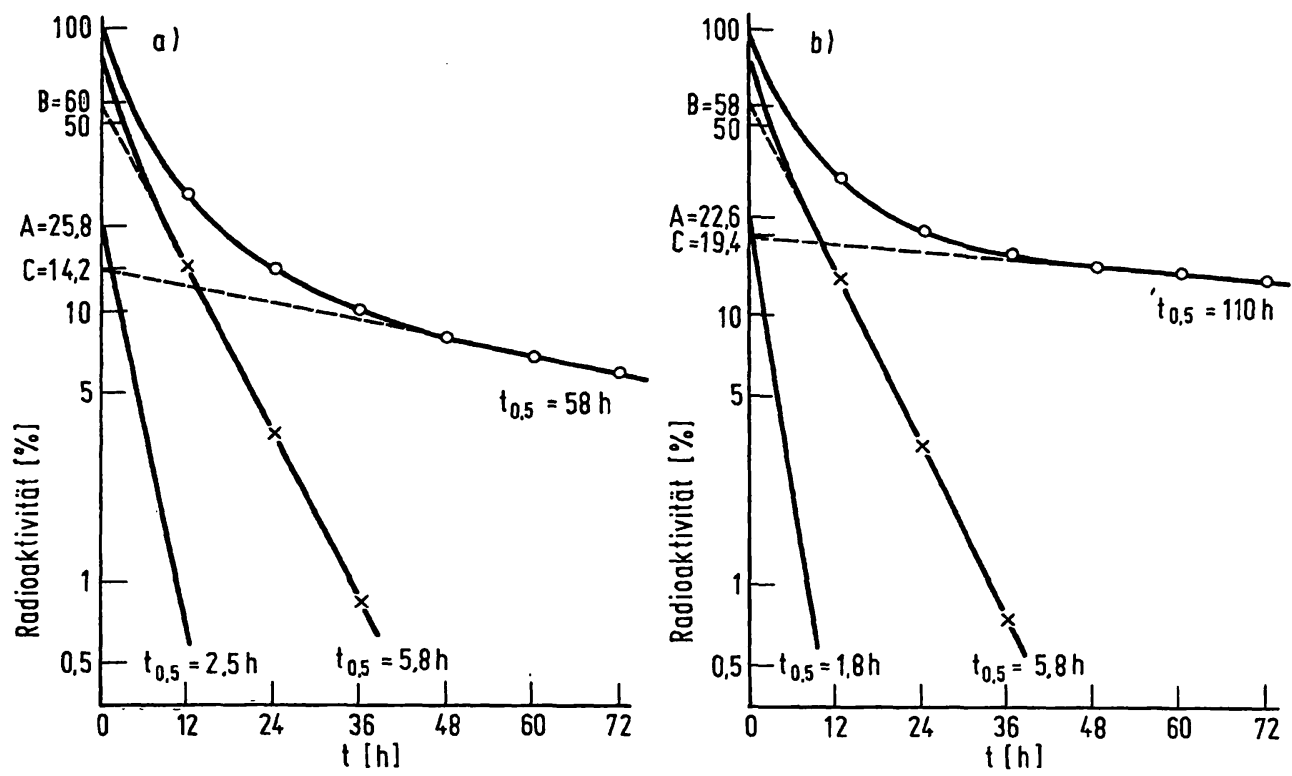

Abb. 11

Die graphische Analyse der 'H-Eliminationskurven aus Abbildung 10 in halblogarithmischer Darstellung ergab als Kurvenfunktion jeweils eine Summe aus 3 negativen Exponentialfunktionen: Radioaktivität $(R a)=A \cdot e^{-x t}+B \cdot e^{-\beta t}+C \cdot e^{-\gamma_{t}}$

Jede dieser drei Einzelfunktionen entspricht einer ${ }^{3} \mathrm{H}$-Fraktion der Größe A, B oder $C(\%)$, die entsprechend einer Geschwindigkeitskonstanten $\alpha, \beta$ oder $\gamma(1 / \mathrm{h})$ über die Nieren eliminiert wird.

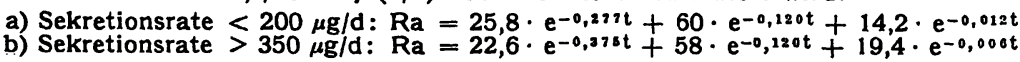

\section{Literatur}

1. Merits, I. (1962), J. Lipid Res. 3, 126-127. - 2. RAPP, J. P. \& EIK-NEs, K. B. (1965), J. Gas Chromatogr. 3, 235-237. 3. RApP, J. P. \& EIK-Nes, K. B. (1966), Anal. Biochem. 15, 386-408. - 4. Bravo, E. L. \& Travis, H. R. (1967), J. Clin. Lab. Med. 70, 831-840. - 5. Salokangas, A. \& Adlercreutz, H. (1967), Acta Endocr. Suppl. 119, 97. - 6. AakvaAg, A. (1967), Acta Endocr. Suppl. 119, 96. - 7. Nicolis, G. L., Worrz, H. H. \& Gabrilove, L. (1968), J. Clin. Endocrin. Metab. 28, 547-557. 8. Fabre, L. F. jr., Fenimore, D. C., Farmer, R. W., Davis, H. W. \& FarrelL, G. (1969), J. Chromatogr. Sci. 7, 632-638. 9. Palem-Vliers, M., Lapiere, C. L. \& Genard, P. (1970), Excerpta Medica Foundation, Intern. Congr. Ser. 210, 99. 10. Leung, F. Y. \& Griffiths, J. (1972), Clin. Chim. Acta 37, 423 - 432. - 11. LOMMER, D. unveröffentlicht. - 12. VECSEI, P. \& LOMMER, D. unveröffentlicht. - 13. NowaCzYnskr, W. J., Gold-
NER, M. \& Genest, J. (1955), J. Clin. Lab. Med. 45, 818-821. 14. Glaz, E. \& VeCSEI, P. (1971), Aldosterone. S. 29-32, Akadémiai Kiadó, Budapest (joint edition with Pergamon Press, Oxford 1971). - 15. Mayes, D., Furuyama, S., Kem, D. C. \& Nugent, C. A. (1970), J. Clin. Endocrinol. Metab. 30, 682-685. 16. Bayard, F., Beitins, I. S., Kowarski, A. \& Migeon, C. J. (1970), J. Clin. Endocrinol. Metab. 31, 507-510. - 17. Katz, F. H., Simet, B. S., Zimering, B. S. \& Kelly, W. G. (1970), Proc. 52nd Mtg. Endocr. Soc. 54, 63. - 18. FARMER, R. W., Roup, W. G. jr., Pellizzari, E. D. \& Fabre, L. F. jr. (1972), J. Clin. Endocrinol. Metab. 34, 18-22. - 19. Vetrer, W., Freedlender, E. \& Haber, E. (1972), Acta Endoct. Suppl. 159, 32. - 20. Neher, R. (1962), Symp. Dt. Ges. f. Endokrinol. 9, 21-39.
Prof. Dr. D. Lommer I. Medizinische Klinik und Poliklinik der Johannes Gutenberg-Universität 65 Mainz Langenbeckstr. 1 